\title{
AHRQ Update
}

\section{Emergency Departments in Crisis: Opportunities for Research}

Carolyn M. Clancy

"If you can barely get through the night's 911 calls, how on earth can you handle a disaster?" asks Arthur L. Kellerman, M.D., a prominent emergency physician from Emory University.

Although large-scale events such as the September 11 attacks and Hurricane Katrina have heightened awareness and concerns about the ability of our emergency system to handle large-scale events, it is clear that emergency rooms in the country are barely able - and in many cases unable-to handle current, every day demand for their services.

Any one who has been to an emergency department recently can attest to the long waits for care. This anecdotal view is borne out by 2004 data from the Centers for Disease Control and Prevention (McCaig and Nawar, 2006):

- One-fifth of the nation's population had made one or more emergency department visit within the previous 12 months.

- Approximately 10 percent of all ambulatory medical care visits in this country occurred in emergency departments.

- There were about 209 emergency department visits every minute across the United States (Machlin, 2006).

- From 1994 to 2004, the rate emergency departments use increased by 6 percent, from 36.0 to 38.2 visits per 100 persons.

Data from AHRQ's National Healthcare Quality Report (2006) adds to the picture of emergency care in the United States:

- In 2004, 1.8 percent of emergency department patients left before being seen. 
- In 2004, about a quarter of patients admitted to the hospital from an emergency department spent more than 6 hours in the emergency room.

In recent reports, the Robert Wood Johnson Foundation has described hospital emergency departments as "bursting at the seams," while the Institute of Medicine (IOM) entitled one of three recent reports on emergency care in the United States Hospital-Based Emergency Care: At the Breaking Point (IOM, 2006).

In this commentary, I would like to describe some recent findings of this IOM report, highlight some existing AHRQ activities in this area, and discuss some research questions posed by the IOM and which the readers of this journal might consider pursuing. As the leading health services researchers in the world, the readers of $H S R$ can contribute greatly to the body of research that will inform efforts to improve the quality, safety, efficiency, and effectiveness of emergency room care.

\section{THE IOM'S TAKE ON EMERGENCY SERVICES}

Those of us who deliver or study health care services have been well aware that a crisis in emergency care has been looming on the horizon. The signs have been pretty clear: the number of emergency department visits is escalating and ambulance diversions, "boarding," and other supply problems have become an every day occurrence.

In June 2006, the IOM issued three critical reports that examine emergency medical services and emergency care for children (IOM, 2006a, b, c). The IOM panel characterized emergency care in the United States as "overburdened, underfunded, and highly fragmented." AHRQ was very pleased to help fund these vital reports and a series of workshops to aid in the reports' dissemination and hopefully, the implementation of their findings. The reports and fact sheets on the detailed findings are available on the IOM website at http://www.iom.edu/CMS/3809/16107/35010.aspx.

The IOM reports were in accord with the National Report Card on the State of Emergency Medicine issued by the American College of Emergency Physicians in January 2006. ACEP rated each state on a set of 50 criteria, and

Address correspondence to Carolyn M. Clancy, M.D., Director, Agency for Healthcare Research and Quality, John M. Eisenberg Building, 540 Gaither Road, Rockville, MD 20850. 
then gave an overall grade of $\mathrm{C}-$ to the nation as a whole. No state scored grades of either $\mathrm{A}$ or $\mathrm{F}$, but more than 80 percent of states earned mediocre or poor grades, ranging from $\mathrm{C}+$ to $\mathrm{D}$.

Compounding these difficulties, emergency departments are generally under funded. There are many contributing factors, including the high percentage of visits by uninsured patients (16 percent), many of whom cannot pay for care; Medicaid-related patients (22 percent), whose care is reimbursed at low rates in many states; and Medicare patients (15 percent), whose care is reimbursed through a prospective payment system.

The IOM reports present an action agenda for improving emergency care in the United States. The reports include the following recommendations:

- Develop a coordinated, regionalized, accountable system. All components of the emergency care system (from emergency medical services to emergency departments) should fully coordinate their activities and integrate communications to ensure seamless emergency services.

- Create a lead federal agency, consolidating all functions related to emergency care.

- End emergency department boarding and diversion. Expanding the capacity of emergency departments is not sufficient; in addition, hospitals should improve operational efficiency and patient flow. The Joint Commission on Accreditation of Healthcare Organizations should reinstate strong standards in this area, and the Centers for Medicare and Medicaid Services should develop incentives to discourage boarding and diversion. Identifying effective strategies to improve hospital efficiency is a priority for AHRQ.

- Increase funding for emergency care. Congress should initially appropriate $\$ 50$ million for hospitals that provide large amounts of emergency care. Funding should also be increased for emergency preparedness efforts of both emergency medical services providers and emergency departments.

- Enhance emergency care research. Federal agencies should increase research funding for prehospital emergency medical services and pediatric emergency care, and the Department of Health and Human Services should assess research needs in emergency care.

- Promote workforce standards for emergency medical services. There should be national accreditation of paramedic education, state acceptance of 
that certification for licensure, and adoption of common emergency medical services certification levels.

- Enhance pediatric presence throughout emergency care. Emergency departments and emergency medical services should employ pediatric coordinators; pediatric concerns should be explicitly addressed in disaster planning; more research should focus on pediatric emergency care; and Congress should increase annual funding for the Emergency Medical Services for Children Program to \$37.5 million for 5 years.

\section{AHRQ'S EMERGENCY SERVICES RESEARCH}

Improving the safety, quality, efficiency of, and access to, emergency services is not a new area of study for AHRQ; we have invested in the research of emergency medical services for many years. Given the depth and breadth of AHRQ activities related to emergency care, I can only touch on a few highlights that exemplify our work in this important area.

In 2001, an AHRQ grant supported the development of the multiinstitutional and multidisciplinary Center for Safety in Emergency Care. This consortium of the University of Florida, Dalhousie University, Northwestern University, and Brown University served as a research center on patient safety in emergency care. There were four areas of primary concern to the consortium:

- the cognitive psychology of human behavior and error,

- the clinical epidemiology of adverse events,

- the use of technology to improve performance and reduce errors, and

- the application of human factors engineering to improve safety.

This team subsequently developed research proposals-that received funding from other sources-and published journal articles and book chapters. One publication detailed a pilot study on shift changes that demonstrated that while patient handoffs were associated with medical errors, in many cases an impending error was averted through the exchange of information (Perry 2004).

Since 1999, as part of AHRQ's Healthcare Cost and Utilization Project, the State Emergency Department Databases has provided de-identified 
patient encounter data from 23 states for more than 100 clinical and nonclinical variables, such as diagnostic and procedure information, patient demographics, total charges, and expected payment sources. AHRQ encourages greater use of this database by researchers to identify trends and develop strategies that enhance emergency care services.

The State Emergency Department Databases are available at http:// www.hcup-us.ahrq.gov/seddoverview.jsp\#How

The IOM reports underscored the need for improving patient flow in hospitals, beginning in the emergency department. One of our long-standing projects is the Emergency Severity Index (ESI) (Gilboy et al. 2005). ESI is a five-level emergency department triage algorithm that yields rapid, replicable, and clinically relevant stratification of patients into five groups; from 1 (most urgent) to 5 (least urgent) on the basis of patient acuity and resource needs. Since the publication of the first edition in the late 1990s, research has led to further refinement of the algorithm. In June 2005 we issued ESI version 4.

One of AHRQ's more promising current investments is a 5-year grant to HealthPartners Research Foundation in Minneapolis, entitled "Emergency Department Crowding: Causes and Consequences." The principal investigator, Brent Asplin, M.D., Department Head of Emergency Medicine at Regions Hospital in St. Paul, also served as a member of the IOM Committee on the Future of Emergency Care.

Brent Asplin also served primary investigator on an AHRQ-sponsored project which resulted in a diverse national panel of experts proposed measurement sets to monitor ED crowding.

This project will provide much needed empirical work that rigorously described the problem of emergency department overcrowding and will propose effective solutions. To date, this grant has already yielded several journal articles that are making important contributions to research and discussions in this area. AHRQ has also made significant investments in information technology that improves the safety and quality of emergency department care. For example, as part of a 3-year grant, the Louisiana Rural Health Information Technology Partnership will implement and evaluate the impact of a computerized medical record in the emergency departments of 17 facilities in their critical access hospital network. The technology is expected to improve information exchange and continuity of care by documenting all elements of an emergency visit.

The research and resources described here represent just a sample of AHRQ's many investments in emergency care research. Other recent AHRQ publications and projects have documented the racial and ethnic disparities in 
emergency care and the wide variation in psychiatric emergency services in emergency departments.

\section{RESEARCH QUESTIONS}

Each of the three IOM reports offers a number of intriguing research questions and opportunities that would help to build the foundation of evidence for emergency medical and department services. Overall, two overarching recommendations aim to expand the research base in this area.

First, the IOM made a recommendation to the private sector: "Academic medical centers support emergency and trauma care research by providing research time and adequate facilities for promising emergency care and trauma investigators, and by strongly considering the establishment of autonomous departments of emergency medicine."

The IOM also suggested that "The Department of Health and Human Services should conduct a study to examine the gaps and opportunities in emergency and trauma care research, and recommend a strategy for the optimal organization and funding of the research effort."

There are currently ongoing discussions within HHS about this recommendation.

For its part, supporting research on emergency medical services remains an important goal for AHRQ. We recently posted a special emphasis notice highlighting our research priorities as they relate to organizational systems: http://grants.nih.gov/grants/guide/notice-files/NOT-HS-07-045.html.

In December, we posted four funding announcements that would provide up to $\$ 26$ million in new research projects to improve the safety and quality of ambulatory health care, which includes emergency department services. The projects funded under these announcements would promote the use of health information technology to improve quality and safety, which is consistent with the IOM reports' recommendations. Information is available at http://www.ahrq.gov/fund/grantix.htm.

The following is a sample of the important research questions and information gaps raised in the IOM reports. I urge you to review the full reports to see a more complete list of research ideas. AHRQ is reviewing the reports to shape future funding announcements that could be made available as our budget allows. Funding announcements will be publicized through AHRQ's Electronic Newsletter. If you do not currently subscribe, go to http:// www.ahrq.gov/news/ahrqlist.htm to sign up. 
1. The impact on emergency department crowding of bottlenecks in different hospital units (ICU, telemetry, etc.) on patient flow.

2. The effectiveness of queuing theory in smoothing of patient volume on crowding, boarding and diversion.

3. The causes and solutions for missed diagnosis in the emergency department.

4. Validation of using prehospital 12-lead electrocardiography to direct patients with acute STEMI to interventional cardiac centers.

5. Evaluation of safe alternatives to endotracheal intubation to secure the airway in the prehospital and emergency department setting.

6. How safe is pediatric emergency care?

7. How effective is pediatric emergency care?

8. How patient-centered is pediatric emergency care?

9. How efficient and timely is pediatric emergency care?

10. How equitable is pediatric emergency care?

\section{CONCLUSION}

Ensuring the availability, efficiency, and safety of emergency services is a national imperative. This is critical not because of potential for a large-scale disaster, but for the millions of patients who visit emergency departments each year care. AHRQ is committed to enhancing and assuring the quality and safety emergency services in this Nation.

\section{REFERENCES}

American College of Emergency Physicians. 2006. "National Report Card on the State of Emergency Medicine.” Available at http://my.acep.org/site/PageServer? pagename=wp1_state_national

Gilboy, N., P. Tanabe, D. A. Travers, A. M. Rosenau and D. R. Eitel. 2005. Emergency Severity Index, Version 4: Implementation Handbook. AHRQ Publication No. 05-0046-2. Rockville, MD: Agency for Healthcare Research and Quality. Available at http://www.ahrq.gov/research/esi

Institute of Medicine (IOM). 2006a. Hospital-Based Emergency Care: At the Breaking Point. Washington, DC: National Academy Press.

- 2006b. Emergency Medical Services at the Crossroads. Washington, DC: National Academy Press.

—. 2006c. Emergency Care for Children: Growing Pains. Washington, DC: National Academy Press. 
Machlin, E. R. 2006. Expenses for a Hospital Emergency Room Visit 2003. Statistical Brief \#111. Rockville, MD: Agency for Healthcare Research and Quality.

McCaig, L. F., and E. W. Nawar. 2006 National Hospital Ambulatory Medical Care Survey: 2004 Emergency Department Summary. National Center for Health Statistics (NCHS). Advance Data from Vital and Health Statistics, No. 372, June 23, 2006.

National Healthcare Quality Report. 2005. U.S. Department of Health and Human Services, Agency for Healthcare Research and Quality, Rockville, MD, AHRQ Publication No. 06-0018, December 2005. Available at: http://www.ahrq.gov/ qual/nhqr05/nhqr05.pdf

Perry, S. 2004. "Transitions in Care: Studying Safety in Emergency Department Signovers." Focus on Patient Safety 7 (2): 1-3. 